\title{
LA MEMBRANA DE LA PUNTEADURA EN DOS ESPECIES DE Cacteae, Cactaceae
}

\author{
Dalia Grego-Valencia ${ }^{1,4}$ Teresa Terrazas ${ }^{2}$, Reyna Lara-Martínez ${ }^{3}$ \\ y LUIS FELIPE JIMÉNEZ-GARCÍA ${ }^{3}$ \\ 'Unidad de Morfología y función, Facultad de Estudios Superiores Iztacala, Universidad Nacional autónoma de México, \\ Tlalnepantla, Estado de México, México \\ ${ }^{2}$ Instituto de Biología, Universidad Nacional Autónoma de México, México, D.F, México \\ ${ }^{3}$ Facultad de Ciencias, Departamento de Biología Celular, Universidad Nacional Autónoma de México, \\ México, D.F., México \\ ${ }^{4}$ Autor para correspondencia: dagreval@gmail.com
}

\begin{abstract}
Resumen: En este trabajo se caracterizan y comparan las punteaduras, la pared secundaria y la membrana de la punteadura en elementos traqueales en dos especies de Cactaceae con madera contrastante. Se recolectaron individuos adultos de Echinocactus grusonii (madera dimorfa) y Mammillaria compressa (madera no fibrosa), pertenecientes a la tribu Cacteae, y se procesaron utilizando diferentes métodos para su observación por microscopía fotónica, electrónica de barrido y de transmisión. Los elementos de vaso tienen placas de perforación simples con un reborde, así como diferentes grados de acumulación de la pared secundaria, desde anulares y helicoidales hasta punteaduras pseudoescalariformes y alternas. En $M$. compressa la pared secundaria en algunos elementos de vaso puede acumularse tanto en forma anular como helicoidal. En E. grusonii la membrana de las punteaduras alternas en los elementos de vaso tiene poros muy pequeños, apreciables exclusivamente a grandes aumentos con microscopia electrónica. En las fibras se observaron organelos, núcleo y mitocondrias, así como un material electrodenso; además, el par de punteaduras fibra-fibra tiene un pequeño reborde, visto con microscopia electrónica de transmisión, no descrito para la familia. Las diferencias en la membrana de la punteadura entre ambas especies posiblemente están relacionadas con su capacidad de evitar embolismos, lo que se podrá demostrar con trabajo empírico.
\end{abstract}

Palabras clave: Cactaceae, elementos de vaso, fibras, membrana de la punteadura, pared secundaria, punteadura, traqueidas de banda ancha, xilema secundario.

\begin{abstract}
In this paper pits, secondary wall, and pit membrane of tracheary elements of two species of Cactaceae with contrasting wood were characterized and compared. Mature plants of Echinocactus grusonii (dimorphic wood) and Mammillaria compressa (non-fibrous wood) belonging to the tribe Cacteae were collected and prepared using different methods to study them through photon, scanning electron, and transmission electron microscopy. Vessel elements have simple perforation plates with a border, as well as different degrees of secondary wall accumulation from annular to helical up to pseudoscalariform and alternate pits. In $M$. compressa secondary wall in some vessel elements may accumulate both annular and helical secondary wall. In E. grusonii pit membrane in the alternate pits of the vessel elements has very small pores, seen only at high magnification with electron microcopy. In fibers, we observed organelles, nucleus, and mitochondria, as well as an electron dense material; furthermore, the pits pair fiber-fiber has a small edge, seen with transmission electron microscopy, not described previously for the family. The pit membrane differences found between species may be related to their ability to avoid embolism, thus empiric research is needed to demonstrate it.
\end{abstract}

Keywords: Cactaceae, fibers, pit, pit membrane, secondary wall, secondary xylem, vessel elements, wide-band tracheids.

L as paredes celulares secundarias comúnmente se caracterizan por la presencia de cavidades llamadas punteaduras, mientras que la lámina media y las dos paredes primarias entre dos punteaduras son llamadas "membrana de la punteadura" (Evert, 2006). Zimmerman y Brown (1971) argumentan acerca del papel fundamental que desempeñan las punteaduras para mantener el flujo de agua continuo en las plantas, ya que además del transporte de agua, protegen al sistema conductor de agua, de la propagación de los embolismos o de los patógenos (Choat et al., 2008). Trabajos 
recientes de fisiología han demostrado que el 50\% de la resistencia hidráulica se debe a las características de la membrana de las punteaduras (Hacke et al., 2006). Sin embargo, el trabajo estructural que permite entender el arreglo de las microfibrillas de celulosa se ha estudiado principalmente en coníferas (Liese y Fahnenbrock, 1952; Dute et al., 2008) y en pocas angiospermas de bosques templados (Jansen et al., 2009). De acuerdo a estos autores, la membrana de las punteaduras en las especies analizadas muestra diferencias en su grosor y en su porosidad y, ambas están relacionadas con el diámetro de la punteadura y su capacidad de cavitar (Jansen et al., 2009); pero, ¿qué ocurre en especies con tallos suculentos que están expuestos a condiciones de estrés hídrico, tienen el mismo tipo de porosidad en las membranas de las punteaduras de sus vasos que otras especies no suculentas?

La familia Cactaceae es un sistema de estudio apropiado para caracterizar las membranas de las punteaduras debido a que esta familia tiene en los elementos de vaso diferentes grados de acumulación de la pared secundaria. Por ejemplo, en las especies con maderas fibrosas, como Pereskia, Pachycereus, Pilosocereus y Polaskia, los elementos de vaso siempre tienen un esqueleto completo de pared secundaria, punteadura o punteado escalariforme, pseudoescalariforme o alterno, mientras que las especies con maderas no fibrosas como Ariocarpus, Coryphantha, Mammillaria y Melocactus, tienen elementos de vaso con engrosamientos de pared secundaria que varían de anulares y helicoidales hasta reticulares. El objetivo de este trabajo fue comparar las características de las punteaduras, la pared secundaria y la membrana de la punteadura, en elementos traqueales de Echinocactus grusonii, con madera dimorfa, y Mammillaria compressa, con madera no fibrosa, sensu Mauseth y Plemons-Rodriguez (1998) y, Terrazas y Mauseth (2002), uti- lizando tres tipos diferentes de microscopía, la microscopía fotónica (MF), la microscopía electrónica de barrido (MEB) y la microscopía electrónica de transmisión (MET).

\section{Materiales y métodos}

Se recolectaron individuos adultos, pertenecientes a la tribu Cacteae, de la especie Echinocactus grusonii $(80 \mathrm{~cm}$ de alto) del Jardín Botánico de la Facultad de Estudios Superiores Zaragoza, y de Mammillaria compressa $(18 \mathrm{~cm}$ de alto), colectada en Estación Ventura, San Luis Potosí. Para ambas especies se separó el cilindro vascular en la base del tallo por ser la región con mayor acumulación de xilema secundario. En el caso de E. grusonii se separaron dos fascículos de médula a cámbium vascular de $1 \mathrm{~cm}$ de alto y, para $M$. compressa, la mitad del cilindro vascular también de $1 \mathrm{~cm}$ de alto (Figura 1). El material se fragmentó en diferentes tamaños según la técnica a utilizar. Para MF y MEB, el cilindro se fragmentó en secciones de $1 \mathrm{~cm}^{3}$ aproximadamente y para MET en secciones menores a $1 \mathrm{~mm}^{3}$. Las muestras para MF y MEB fueron fijadas en FAA (Formol, ácido acético glacial, alcohol etílico) (Johansen, 1940) por 24 horas.

Microscopía fotónica. Después de la fijación, los fragmentos se deshidrataron en concentraciones crecientes de alcohol terbutílico con inmersiones cada $24 \mathrm{~h}$ en un procesador de tejidos marca LeicaTP1020 (Alemania). Posteriormente, las muestras se incluyeron en parafina Histowax (Leica) con punto de fusión de $56-58{ }^{\circ} \mathrm{C}$. Se realizaron cortes transversales, tangenciales y radiales de aproximadamente 15-20 $\mu \mathrm{m}$ con un micrótomo rotatorio marca Leica RM2125 (Alemania) y se tiñeron con safranina-verde rápido (Johansen, 1940). Finalmente se montaron en resina sintética.

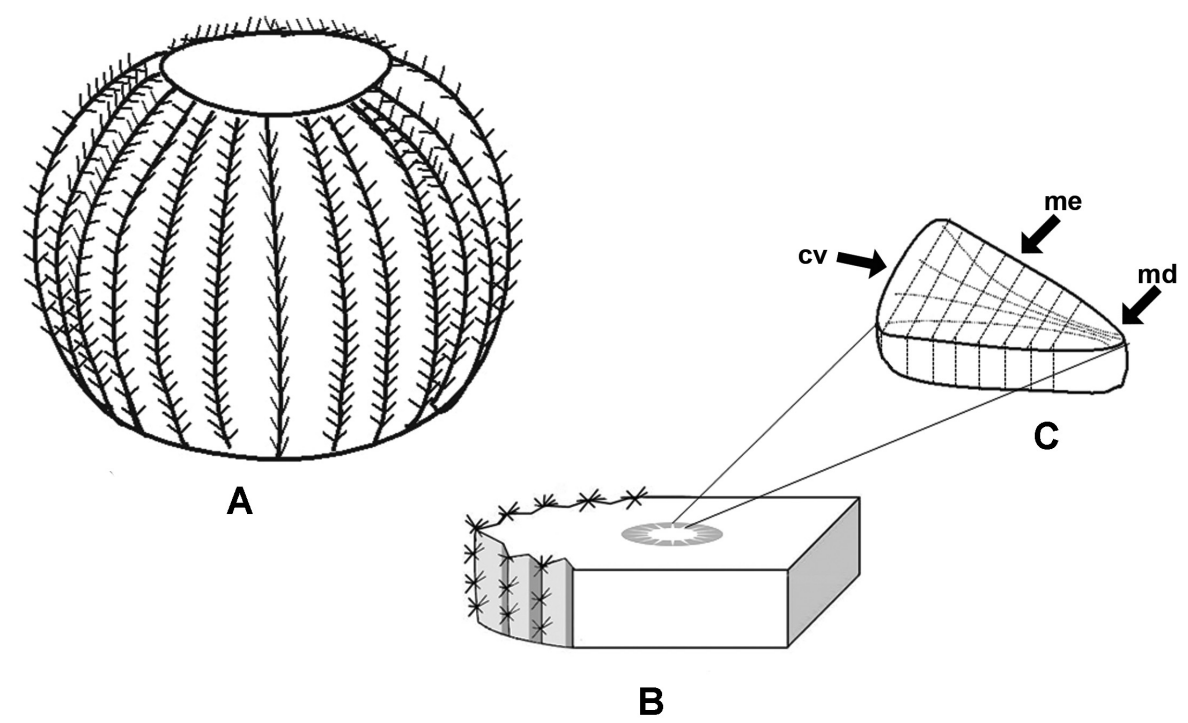

Figura 1. Diagrama que ilustra la forma de colecta del tejido vascular. A) Forma de crecimiento cilíndrica. B) Sección transversal del tallo, su parte distal. C) Fascículo vascular para las microscopias. cv = cámbium vascular, me = médula, md = media. 
Microscopía electrónica de barrido. Después de la fijación, los fragmentos de madera se lavaron con agua destilada. Se realizaron cortes transversales y tangenciales, con un micrótomo de deslizamiento marca Leica 2000R (Alemania) a $80 \mu \mathrm{m}$, se deshidrataron en concentraciones crecientes de etanol del $50 \%$ al $100 \%$ por $24 \mathrm{~h}$ en cada uno; posteriormente se secaron en un horno a $60{ }^{\circ} \mathrm{C}$ durante un día. Los cortes ya secos se montaron en un portamuestras de aluminio utilizando cinta de carbón de doble cara y se recubrieron con oro en un ionizador marca Quórum modelo 150RES (Japón). El análisis de las muestras fue realizado en un microscopio electrónico de barrido marca HITACHI modelo S-2460 (Japón).

Microscopia electrónica de transmisión. Se obtuvo material fresco de Echinocactus grusonii, se fijó con glutaraldehído al $6 \%$ y paraformaldehído al $4 \%$, durante 90 min. La muestra de Mammillaria compressa se fijó con FAA, debido a que proviene de una colecta realizada previamente y guardada en la colección del Laboratorio de Botánica Estructural del Instituto de Biología. De los fascículos de ambas especies se eligieron fragmentos de la madera de la región más cercana a cámbium vascular y para Echinocactus grussonii también de la región más cercana a médula donde solo hay traqueidas de banda ancha (TBA), y así tener representadas las dos formas de su madera dimorfa (Figura 1C). Posteriormente, el material se lavó con buffer de fosfatos (PBS, $0.1 \mathrm{M} \mathrm{y} \mathrm{pH} \mathrm{7.2),} \mathrm{se}$ postfijó con tetraóxido de osmio al $1 \%$ durante toda la noche (Jiménez-Ramírez et al, 2002) y se lavaron nuevamente con buffer de fosfatos. La deshidratación se realizó con etanol del 30 al 100\%, según Jansen et al. (2009), y la preinclusión se realizó en diferentes concentraciones de óxido de propileno y resina Epon 812, durante $24 \mathrm{~h}$ cada uno, hasta llegar a resina pura. Se polimerizaron en una estufa a $60{ }^{\circ} \mathrm{C}$ por 48 horas. Se obtuvieron cortes semifinos transversales y tangenciales de $300 \mathrm{~nm}$ de grosor con cuchillas de vidrio en un ultramicrótomo Leica Ultracut R (Alemania). Los cortes semifinos fueron teñidos con azul de toluidina y revisados en un microscopio de campo claro modelo Axiostar de Zeiss (Alemania), acoplado a una cámara Canon (Taiwan) y el programa EOS-1000D para la captura y registro de imágenes. También se realizaron cortes ultrafinos de 40 a $60 \mathrm{~nm}$ de grosor que se recogieron en anillos de Sjöstrand, se contrastaron durante 3 min con permanganato de potasio al $1 \%$. Los cortes fueron observados en un microscopio electrónico de transmisión marca JEOL, modelo JEM1010 (Japón) y la captura de las imágenes se realizó con una cámara MTI modelo CCD-300RC (Estados Unidos) y el programa Scion Image. Los términos para describir la membrana de la punteadura se tomaron de Jansen et al. (2009).

\section{Resultados}

Microscopía fotónica. En los cortes transversales de las dos especies estudiadas, se observaron los vasos arreglados en una matriz de fibras o traqueidas de banda, así como células de los radios con paredes primarias (Figuras 2A, B, 3A). En la región cercana a cámbium vascular en Echinocactus grusonii se encuentran los elementos de vaso (EV) embebidos en una matriz de fibras, mientras que en la región cercana a la médula los EV se encuentran embebidos en una matriz de traqueidas de banda ancha (Figura 2A, B). Se observaron las placas de perforación (Figura 2C) de los elementos de vaso, así como los diferentes grados de acumulación de la pared secundaria desde anulares y helicoidales hasta punteaduras pseudoescalariformes (Figura 2D).

En Mammillaria compressa se observaron las traqueidas de banda ancha con los engrosamientos anulares y helicoidales de la pared secundaria. En algunos elementos de vaso la pared secundaria puede acumularse tanto en forma anular como helicoidal (Figura 3B). En los cortes semifinos se observaron las punteaduras vaso-vaso y traqueida de bandatraqueida de banda ancha (Figura 3C, D).

Microscopía Electrónica de Barrido. En Echinocactus grusonii, los elementos de vaso tienen la placa de perforación simple con un reborde (Figura 4A), además en sus paredes laterales presentan engrosamientos helicoidales de la pared secundaria (Figura 4B) o bien las punteaduras pseudoescalariformes y alternas (Figura 4A, C). La membrana de las punteaduras pseudoescalariformes y alternas presentan poros muy pequeños (Figura 4D). En la madera no fibrosa de Mammillaria compressa, los elementos traqueales presentan el mismo patrón de arreglo de la pared secundaria (Figura 5A). La pared primaria se aprecia ligeramente plegada con apariencia rugosa y con tendencia a helicoidal (Figura 5B, C) y la membrana de la punteadura es más frágil y no porosa (Figura 5D).

Microscopía Electrónica de Transmisión. En cortes transversales, de la parte media del haz vascular de Echinocactus grusonii, se registraron punteaduras fibra-fibra (Figura 6A, $\mathrm{B})$, fibra-parénquima (Figura 6C), traqueida de banda ancha-traqueida de banda ancha (Figura 7A, B) y vaso-vaso (Figura 7C). En las paredes celulares se observó un arreglo homogéneo de las capas que las conforman. En los pares de punteaduras fibra-fibra se observó un material electrodenso (Figura 6B), mientras que en el lumen celular de la fibra se aprecian organelos tales como núcleo y mitocondrias (Figura 6D). En el par de punteaduras fibra-parénquima se observó un ligero reborde de la pared secundaria (Figura 6C). En esta especie, la membrana de la punteadura es ligeramente electrodensa y porosa (Figura 7D).

En Mammillaria compressa, los cortes transversales de los elementos traqueales mostraron la pared primaria con canales ubicados a diferente profundidades (Figura 8A, B). En los anillos o hélices de pared secundaria de los elementos traqueales se observan prolongaciones de pared secundaria (Fig. 8C, D). Entre cada engrosamiento de la pared secun- 
daria, en cortes tangenciales, se aprecia la membrana de la punteadura con fibrillas de arreglo irregular (Figura 8E, F).

\section{Discusión}

Las micrografías obtenidas en este estudio confirman las características de los elementos traqueales que han descrito varios autores en la madera de diferentes angiospermas
(Evert, 2006). Además se observan características de los elementos traqueales que no han sido registradas para estas especies de la familia Cactaceae.

Elementos de vaso. Los engrosamientos de la pared secundaria, tanto en elementos de vaso como en traqueidas de banda ancha, también fueron observados como lo han indicado otros autores para especies de la familia Cactaceae

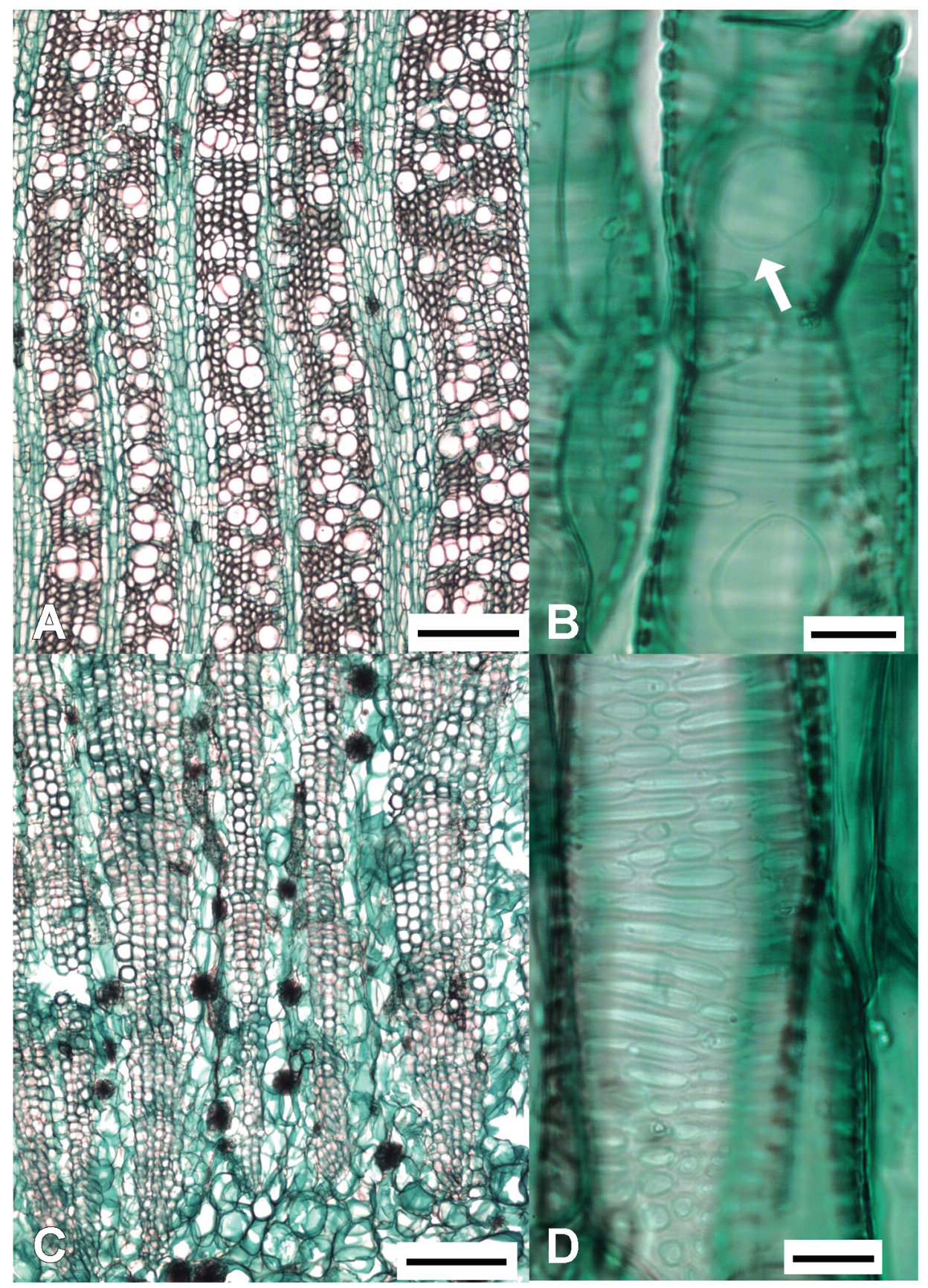

Figura 2. Madera dimorfa en Echinocactus grusonii, cortes transversales (CT) y tangenciales (CTa.). A) Vasos en la matriz de fibras cerca de cámbium vascular. B) Vasos en la matriz de traqueidas de banda ancha cerca de médula. C) Placas de perforación simple en elemento de vaso, CTa. D) Punteaduras pseudoescalariformes en elemento de vaso. Escala: A, B $=300 \mu \mathrm{m} ; \mathrm{C}, \mathrm{D}=20 \mu \mathrm{m}$. 

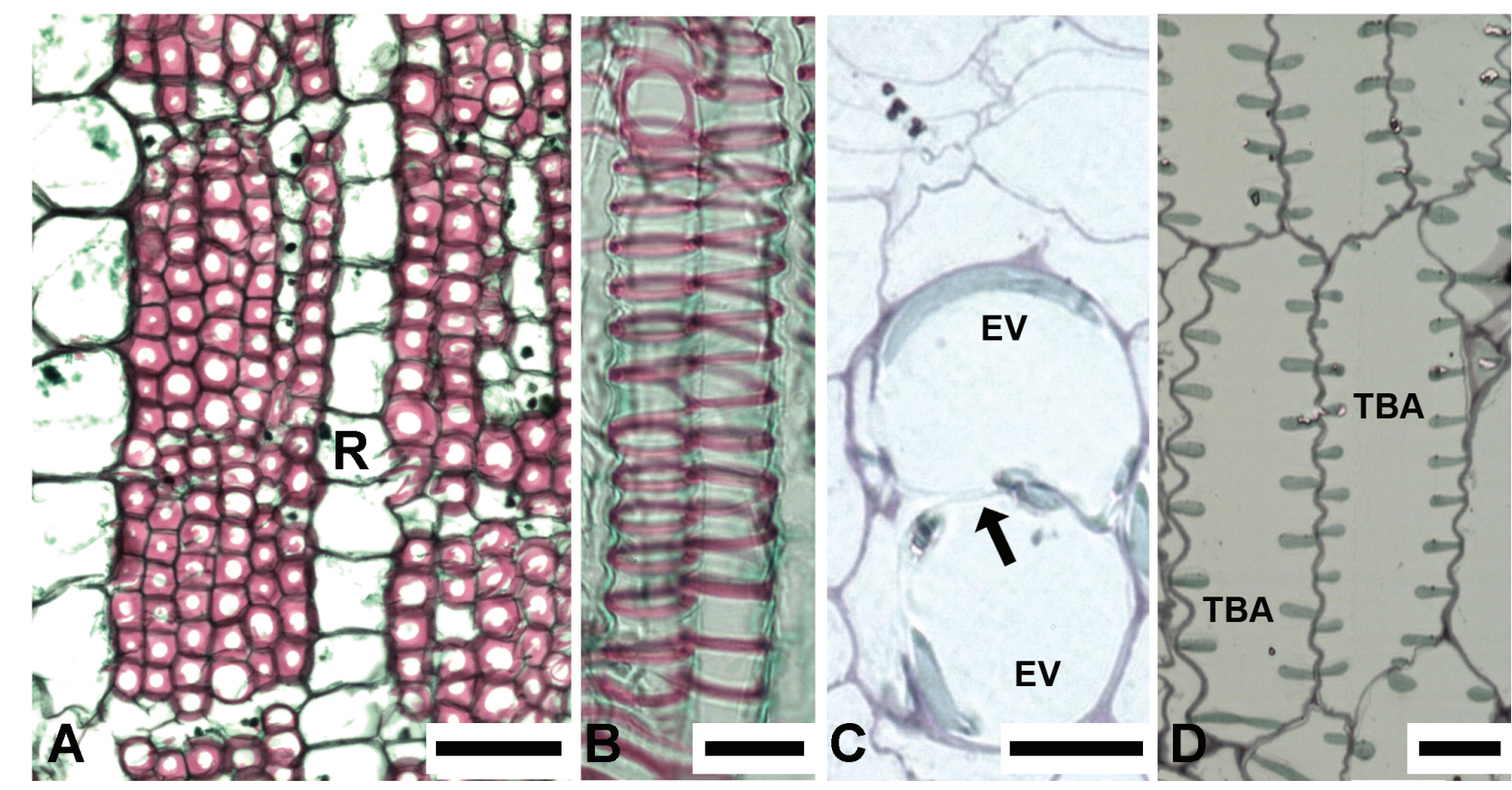

Figura 3. Madera no fibrosa Mammillaria compressa, CT y CTa. A) Traqueidas de banda ancha y vasos, CT. B) Engrosamientos helicoidales y anulares de elementos de vaso, CTa. C) Punteaduras vaso-vaso (flecha), CT. D) Traqueidas de banda ancha, CTa. Escala: A = 100 $\mu \mathrm{m}: \mathrm{B}-\mathrm{D}=20 \mu \mathrm{m}$.

(Mauseth et al., 1995; Carlquist, 2009a; Vázquez-Sánchez y Terrazas, 2011). Sin embargo, aquí se muestra que la menor acumulación de pared secundaria (engrosamientos anulares o helicoidales) se presenta en la asociación elementos de vaso-traqueidas de banda ancha. Cuando los elementos de vaso se encuentran en la matriz de fibras hay una gradación en la acumulación de la pared secundaria de los elementos de vaso hasta tener punteaduras pseudoescalariformes y alternas.

La placa de perforación que se observó es simple, como se ha reportado para otros miembros de Cactaceae (Gibson, 1973; Mauseth y Fujii, 1994; Vázquez-Sánchez y Terrazas, 2011). Además con MEB, se encontró un reborde en la placa de perforación como se registra para algunos géneros de Caryophyllales y, particularmente, en Stenocereus alamosensis de Cactaceae (Carlquist, 2010). Los resultados sugieren que dicho reborde, en la placa de perforación simple, es común en Cactaceae, pero en la actualidad ha sido poco estudiado con MEB.

La pared secundaria en los elementos de vaso presentó engrosamientos del tipo helicoidal en Mammillaria compressa o también helicoidales más punteaduras pseudoescalariformes y alternas en Echinocactus grusonii. Los engrosamientos helicoidales de la pared secundaria y las punteaduras pseudoescalariformes en los elementos de vaso también fueron vistos en otras especies de Cacteae (Vázquez-Sánchez y Terrazas, 2011). Bierhorst y Zamora (1965) también los registran en otras familias de plantas pero en xilema primario e interpretan como una transición de pro- toxilema a metaxilema. Esta interpretación es evidenciada por el trabajo de Falconer y Seagull (1988), quienes observaron que los microtúbulos de elementos traqueales están involucrados en el cambio ontogenético de la depositación de la pared secundaria de protoxilema a metaxilema. Las observaciones en E. grusonii se centran en xilema secundario, producto de cámbium vascular, por lo tanto se infiere que en esta especie, la diversidad del arreglo de la pared secundaria en elementos de vaso no es propia del meristemo de origen (cámbium vascular). Esta deducción se apoya en el trabajo de Loza-Cornejo et al. (2003) donde se observó la transición de engrosamientos helicoidales a punteaduras pseudoescalariformes en los elementos de vaso del xilema primario al secundario en plántulas de Stenocereus queretaroensis y las observaciones en los cortes radiales de ambas especies de médula a cámbium vascular (datos sin publicar). Solo para Gymnocalium cenanthemum, con traqueidas de banda ancha en el xilema secundario, Mauseth et al. (1995) observaron elementos de vaso con punteaduras pseudoescalariformes. Esto no coincide con lo que se observó en $M$. compressa, una especie con madera no fibrosa (madera predominantemente con TBA) donde los elementos de vaso presentan arreglos anulares y helicoidales de la pared secundaria.

Mauseth y Fujii (1994) hallaron en elementos de vaso de Hylocereus bandas lisas y anchas con patrón pseudohelicoidal que representan las áreas entre los engrosamientos de la pared secundaria. Esto no concuerda con lo que se ha encontrado en los elementos de vaso con punteaduras pseu- 

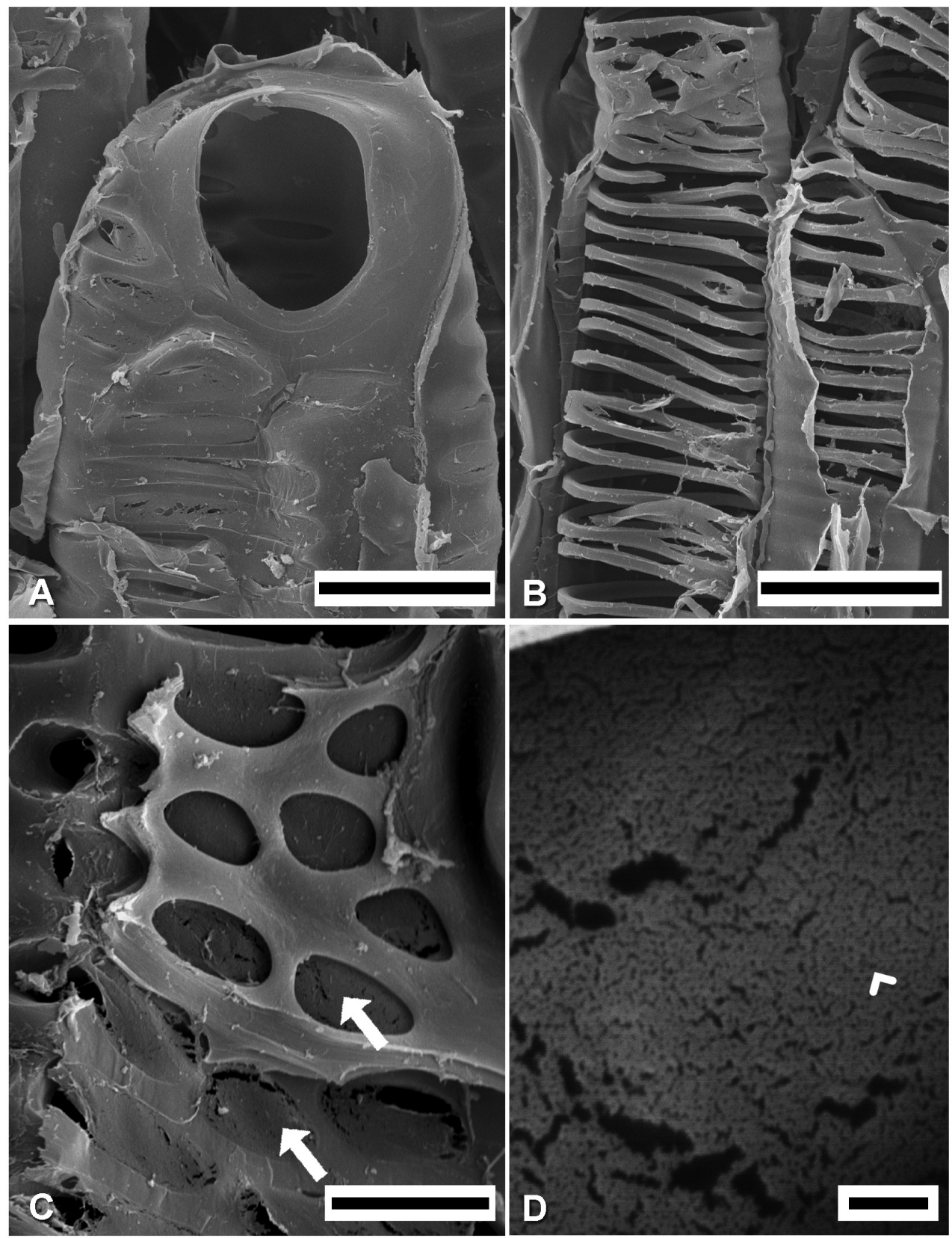

Figura 4. Echinocactus grusonii, corte tangencial (CTa, MEB). A) Placa de perforación simple de EV con reborde y punteaduras pseudoescalariformes. B) Engrosamientos helicoidales de elemento de vaso. C, D) Membrana de la punteadura pseudoescalariformes (flechas), poros (cabeza de flecha). Escala: A, B $=50 \mu \mathrm{m} ; \mathrm{C}=10 \mu \mathrm{m} ; \mathrm{D}=400 \mathrm{~nm}$.

doescalariformes y alternas de Echinocactus grusonii. Con magnificaciones mayores a las que Mauseth y Fujii (1994) utilizaron, sí se observa la membrana de la punteadura con poros pequeños. Estos poros son mucho menores en tamaño con respecto a lo que se ha registrado para otras angiospermas (Jansen et al., 2009), con valores de más de $200 \mathrm{~nm}$. Por lo que para realizar observaciones detalladas de la orientación de microfibrillas en la membrana de la punteadura de estas especies de Cacteae es necesario utilizar un MEB de emisión de campo (Abe et al., 1991).
Traqueidas de banda ancha. La pared secundaria de las TBA en forma anular o de hélice, la han reportado Mauseth et al. (1995), Mauseth (2004) y Carlquist (2009b), para otros géneros de la familia. Aquí se muestra que cuando este tipo de engrosamiento de la pared secundaria se presenta en la TBA, también lo presenta el elemento de vaso. Varios autores mencionan que estos engrosamientos se encuentran separados por la pared primaria (Mauseth et al., 1995; Mauseth, 2004). Esta pared primaria tiene la apariencia de estar arqueada hacia el interior del elemento formando pliegues (Figura 3B, 

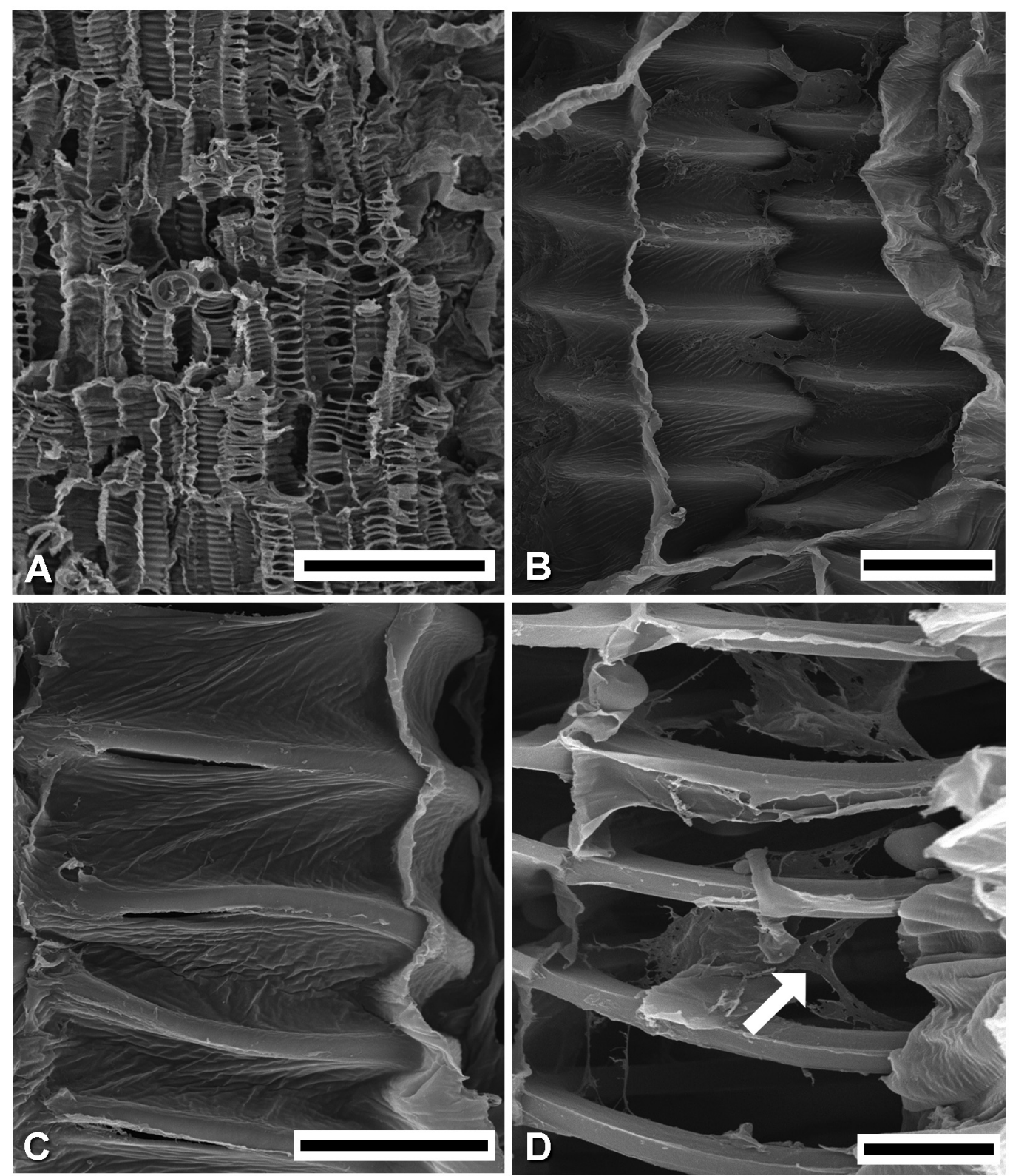

Figura 5. Mammillaria compressa, CTa-MEB. A) Madera no fibrosa, traqueidas de banda ancha TBA. B) Elementos traqueales. C) Pared primaria con pliegues. D) Membrana de la punteadura donde no se aprecian poros a este aumento (flecha). Escala: $\mathrm{A}=200 \mu \mathrm{m} ; \mathrm{B}=25$ $\mu \mathrm{m} ; \mathrm{C}=20 \mu \mathrm{m} ; \mathrm{D}=10 \mu \mathrm{m}$.

C) que indican la elasticidad de la pared primaria gracias al arreglo de microfibrillas de celulosa y otras sustancias pécticas (Esau, 1976). Por lo tanto, si las células pierden agua y se contraen longitudinalmente, las paredes primarias se arquean al interior pero no llegan a cerrar el lumen. Esto se demostró en el trabajo experimental de Garrett et al. (2010) para Ariocarpus fissuratus, especie que también tiene una madera no fibrosa donde predominan las TBA (Vázquez-Sánchez y Terrazas, 2011). Esta capacidad de expansión y contracción de las TBA se relaciona con la ausencia de fibras como en Mammillaria compressa, debido a que ellas impedirían el pliegue de las TBA debido a sus paredes fuertemente lignifi- cadas (Mauseth, 2004; Mauseth, 2006; Vázquez-Sánchez y Terrazas, 2011). Por otro lado, el registro de la pared secundaria anular y helicoidal en un mismo elemento traqueal en $M$. compressa, no había sido observado en Cacteae. Respecto a las TBA, vistas en MEB, no se aprecia una porosidad de la membrana de la punteadura a los aumentos utilizados, como se ha observado en otras familias de plantas para otros elementos traqueales como traqueidas o elementos de vaso (Sano, 2005; Sano y Jansen, 2006; Jansen et al., 2009). Las observaciones, aquí realizadas en MET, permiten apreciar en $M$. compressa un arreglo de fibrillas que forman la membrana de la punteadura, que en MEB se aprecia como no porosa. 

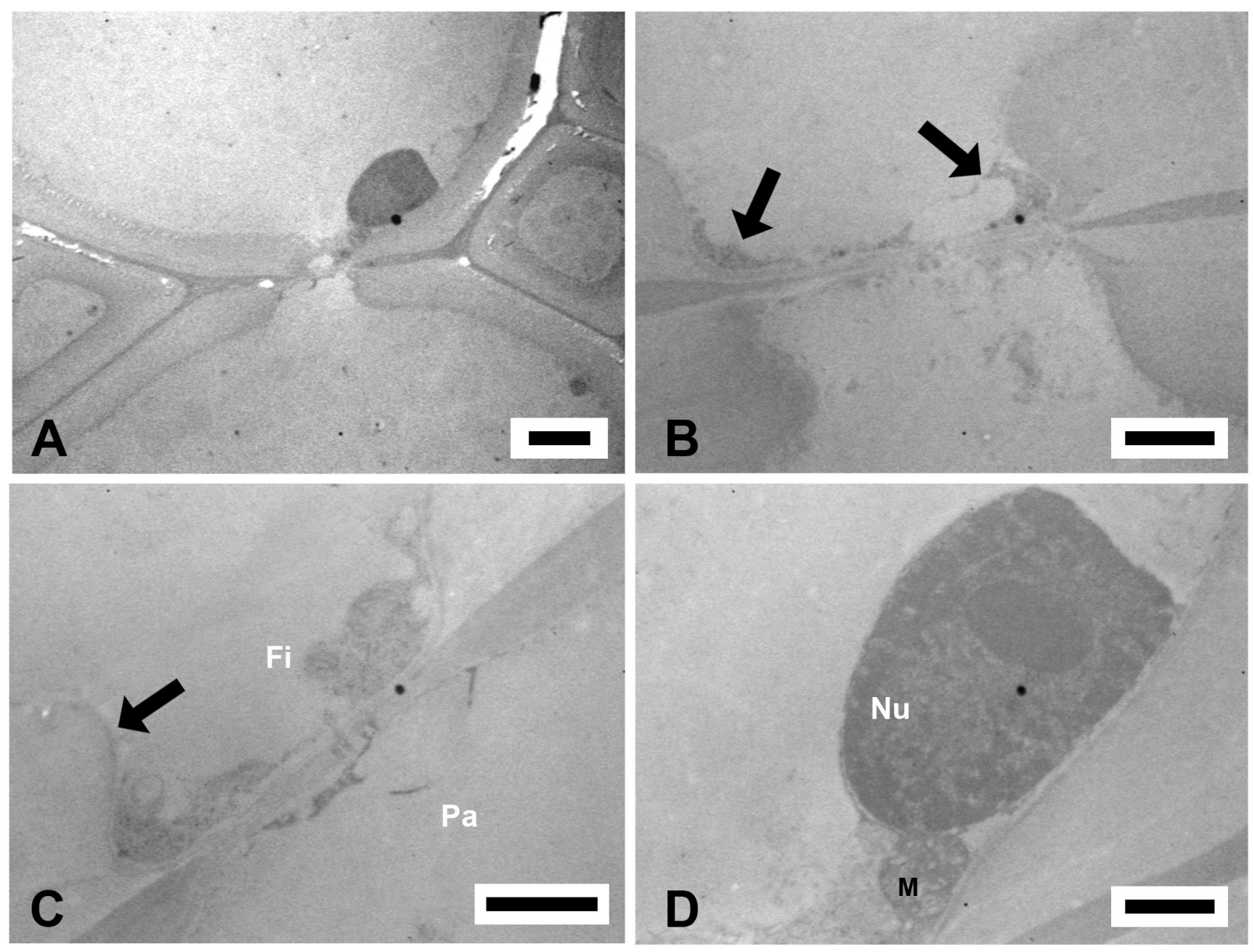

Figura 6. Echinocactus grusonii, CT-MET. A) Punteadura fibra-fibra. B) Membrana de la punteadura fibra-fibra, material electrodenso (flechas). C) Punteadura fibra (Fi)-parénquima ( $\mathrm{Pa}$ ) con ligero reborde (flecha). D) Núcleo (Nu) y mitocondria $(\mathrm{M})$ presente en fibra. Escala: A-D $=1 \mu \mathrm{m}$.

Dicho arreglo se observa (en corte tangencial), entre cada anillo de la pared secundaria. Esto sugiere realizar cortes seriados entre 40 y $80 \mathrm{~nm}$ para lograr tener toda la estructura de la membrana de la punteadura, ya que estudios previos han mostrado que hay múltiples capas de microfibrillas en las membranas de la punteadura con diferentes orientaciones (Schmid y Machado, 1968; Sano, 2005).

Fibras. Con este estudio se confirma que las fibras de Echinocactus son células vivas, como lo reporta Mauseth (2006) para otros miembros de la familia, ya que contienen organelos (núcleo y mitocondrias). Este tipo celular conserva su protoplasto y almacena sustancias como almidón, aceite y resinas, además de conferirle soporte a la planta (Fahn y Leshem, 1963). Su estudio, por primera vez a nivel de MET, confirma que son fibras nucleadas en E. grusonii. La presencia del reborde en el par de punteadura fibra-fibra permite reconocer a las fibras como fibrotraqueidas. Las fibrotraqueidas no han sido reportadas para ningún otro miembro de la familia, se sugiere observarlas en MET para confirmar su presencia en Cacteae o en otras tribus y subfamilias de Cactaceae, ya que el reborde es pequeño y no se distingue a través de la MF. El material electrodenso que se observó en las fibras también lo han reportado para otras especies de eudicotiledóneas (Aesculus hippocastanum, Sorbus aucuparia, Tilia europea) y una especie de gimnospermas (Picea sitchensis, Barnett $e t$ al., 1993). Este material electrodenso se describe como una capa de protección que se encuentra entre el plasmalema y la pared secundaria en una célula de parénquima adyacente a un elemento de vaso, traqueida o fibra.

\section{Conclusiones}

El uso de la microscopía fotónica, microscopía electrónica de barrido y de transmisión, permitió por separado, observar a diferentes aumentos, detalles estructurales de los elementos traqueales y las fibras. Con el uso de las tres técnicas se integra la información obtenida sobre la estructura de la membrana de la punteadura que no se había estudiado en Cacteae. La membrana de la punteadura en Echinocactus grusonii presenta poros solo apreciables a altas magnificaciones, contrario a lo que se ha reportado para otras eudico- 

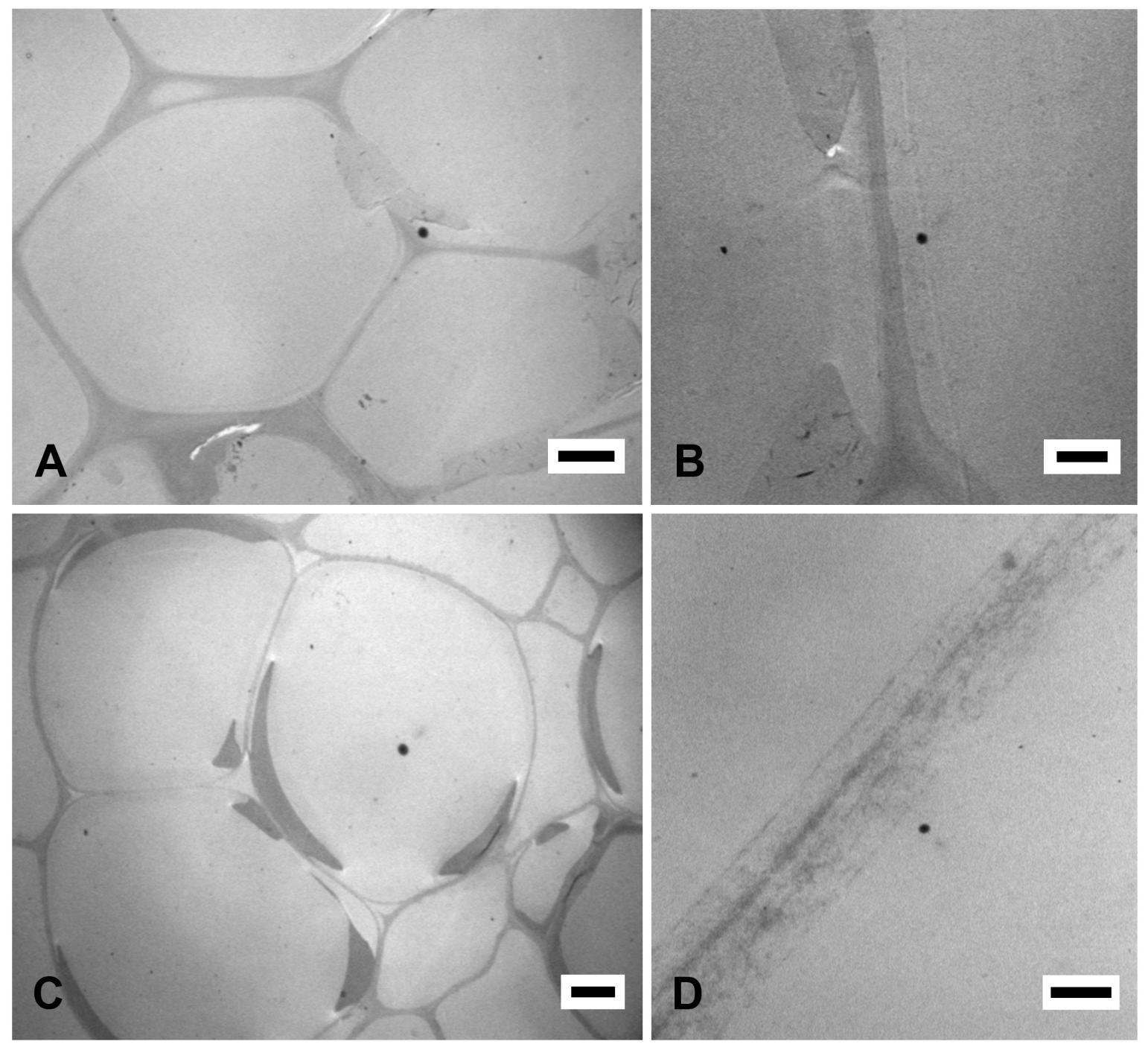

Figura 7. Echinocactus grusonii, CT-MET. A) Vista general de traqueidas de banda ancha. B) Punteadura de traqueidas de banda anchatraqueidas de banda ancha. C) Vista general de vasos. D) Membrana de la punteadura vaso-vaso. Escala: A, C =6 $\mu \mathrm{m}$; B, D = $2 \mu \mathrm{m}$.

tiledóneas y posiblemente esté relacionado con su capacidad de evitar embolismos. La membrana de la punteadura en los elementos traqueales de Mammillaria compressa parece ser más abierta. A medida que se incorpore mayor información en torno a los atributos de las membranas de las punteaduras en Cactaceae se podrán inferir cómo estas especies mantienen el flujo de agua, tanto en sentido vertical como horizontal, conocimiento que se debe complementar con estudios empíricos sobre la arquitectura hidráulica de este grupo de plantas.

\section{Agradecimientos}

Se agradece al Programa de Apoyo a Proyectos de Investigación e Innovación Tecnológica, DGAPA, Universidad Nacional Autónoma de México por el financiamiento al proyecto IN209012. También se agradece a Berenit Mendoza por la ayuda en la toma de electronmicrografias del MEB en el Instituto de Biología, a Julio César Montero Rojas y Diana Ramírez por el diseño fotográfico y a dos revisores anónimos quienes con sus comentarios críticos ayudaron a enriquecer la discusión.

\section{Literatura citada}

Abe H., Ohtani J. y Fukazawa K. 1991. FE-SEM observations on the microfibrillar orientation in the secondary wall of tracheids. IAWA Journal 12:431-438.

Barnett J.R., Cooper P. y Bonner L.J. 1993. The protective layer as an extension of the apoplast. IAWA Journal 14:163-171.

Bierhorst D.W. y Zamora P.M. 1965. Primary xylem elements and element associations of angiosperms. American Journal of Botany 52:657-710.

Carlquist S. 2009a. Non-random vessel distribution in woods: patterns, modes, diversity, correlations. Aliso 27:39-58. 

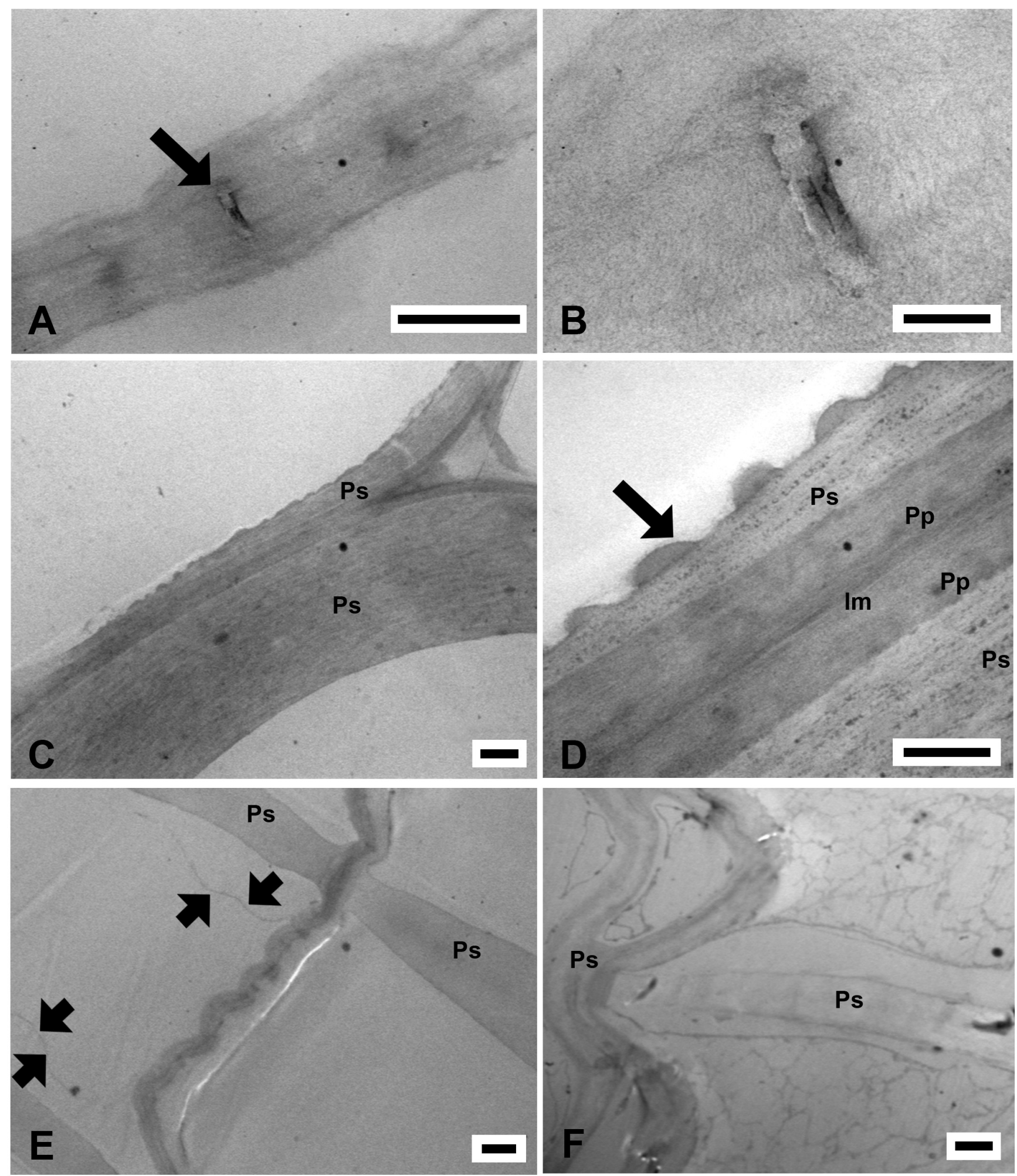

Figura 8. Mammillaria compressa, CT-MET. A) Canal en la pared primaria de elementos traqueales. B) Acercamiento de canal. C) Par de elementos traqueales. D) Extensiones en la pared secundaria de un elemento traqueal, CTa. E) Fibrilla de la membrana de la punteadura elemento traqueal-elemento traqueal (flechas). F) Arreglo irregular de la membrana de la punteadura. $1 \mathrm{~m}=1$ ámina media, $\mathrm{Pp}=\mathrm{pared}$ primaria, Ps $=$ Pared secundaria. Escala: A, C, E, F = $1 \mu \mathrm{m} ; \mathrm{B}=200 \mathrm{~nm} ; \mathrm{D}=0.5 \mu \mathrm{m}$. 
Carlquist S. 2009b. Xylem heterochrony: an unappreciated key to angiosperm origin and diversifications. Botanical Journal of the Linnean Society 161:26-65.

Carlquist S. 2010. Caryophyllales: a key group for understanding wood anatomy character states and their evolution. Botanical Journal of the Linnean Society 164:342-393.

Choat B., Cobb A.R. y Jansen S. 2008. Structure and function of bordered pits: New discoveries and impacts on whole-plant hydraulic function. New Phytologist 177:608-626.

Dute R., Hagler L. y Black A. 2008. Comparative development of intertracheary pit membranes in Abies firma and Metasequoia glyptostroboides. IAWA Journal 29:277-289.

Esau K. 1976. Anatomía Vegetal. Omega, Barcelona.

Evert R.F. 2006. Esau's Plant Anatomy: Meristems, Cells, and Tissues of the Plant Body: their Structure, Function, and Development. 3ra ed., Wiley Interscience, Hoboken.

Falconer M.M. y Seagull R.W. 1988. Xylogenesis in tissue culture III: Continuing wall deposition during tracheary element development. Protoplasma 144:10-16.

Fahn A. y Leshem B. 1963. Wood fibres with living protoplasts. New Phytologist 62:91-98.

Garrett T.Y., Huynh C.V. y North G.B. 2010. Root contraction helps protect the "living rock" cactus Ariocarpus fissuratus from lethal high temperatures when growing in rocky soil American Journal of Botany 97:1951-1960.

Gibson A.C. 1973. Comparative anatomy of secondary xylem in Cactoideae (Cactaceae). Biotropica 5:29-65.

Hacke U.G., Sperry J.S., Wheeler J.K. y Castro L. 2006. Scaling of angiosperm xylem structure with safety and efficiency. Tree Physiology 26:689-701.

Jansen S., Choat B. y Pletsers A. 2009. Morphological variation of intervessel pit membranes and implications to xylem function in angiosperms. American Journal of Botany 96:409-419.

Jiménez-Ramírez J., Agredano-Moreno L.T., Segura-Valdez M.L. y Jiménez-García L.F. 2002. Lacandonia granules are present in Ginkgo biloba cell nuclei. Biology of the cell 94:511-518.

Johansen D.A. 1940. Plant Microtechnique. $5^{\text {a }}$ ed., MacGraw Hill, Nueva York.

Editor Asociado: Silvia Aguilar-Rodríguez

Recibido: 7 de abril de 2014

Aceptado: 9 de septiembre de 2014
Liese W. y Fahnenbrock M. 1952. Elektronenmikroscopische Untersuchungen über den Bau der Hoftüpfel. Holz Roh-Werkstoff 10:197-201.

Loza-Cornejo S., Terrazas T., López-Mata L. y Trejo C. 2003. Características morfo-anatómicas y metabolismo fotosintético en plántulas de Stenocereus queretaroensis (Cactaceae): su significado adaptativo. Interciencia 28:83-89.

Mauseth J.D. 2004. Wide-band tracheids are present in almost all species of Cactaceae. Journal of Plant Research 117:69-76.

Mauseth J.D. 2006. Structure-function relationships in highly modified shoots of Cactaceae. Annals of Botany 98:901-926.

Mauseth J.D. y Fujii T. 1994. Resin-casting: a method for investigating apoplastic spaces. American Journal of Botany 81:104110 .

Mauseth J.D. y Plemons-Rodriguez B.J. 1998. Evolution of extreme xeromorphic characters in wood: a study of nine evolutionary lines in Cactaceae. American Journal of Botany 85:209218.

Mauseth J.D., Uozumi Y., Plemons B.J. y Landrum J.V. 1995. Structural and systematic study of an unusual tracheid type in cacti. Journal of Plan Research 108:517-526.

Sano Y. 2005. Inter- and intraspecific structural variations among intervascular pit membranes, as revealed by Field-Emission Scanning Electron Microscopy. American Journal of Botany 92:1077-1084.

Sano Y. y Jansen S. 2006. Perforated pit membranes in imperforate tracheary elements of some angiosperms. Annals of Botany 97:1045-1053.

Schmid R. y Machado R.D. 1968. Pit membranes in hardwoods - Fine structure and development. Protoplasma 66:185-204.

Terrazas S.T. y Mauseth J.D. 2002. Shoot anatomy and morphology. En: Nobel P.S. Ed. Cacti: Biology and Uses, pp. 23-40, University of California Press, Berkeley.

Vázquez-Sánchez M. y Terrazas T. 2011. Stem and wood allometric relationships in Cacteae (Cactaceae). Trees Structure and Function. 25:755-767.

Zimmerman M.H. y Brown C.L. 1971. Trees: Structure and function. Springer-Verlag, Nueva York. 